\title{
ASYMPTOTICS OF GENERALIZED GALOIS NUMBERS VIA AFFINE KAC-MOODY ALGEBRAS
}

\author{
STAVROS KOUSIDIS
}

(Communicated by Kathrin Bringmann)

\begin{abstract}
Generalized Galois numbers count the number of flags in vector spaces over finite fields. Asymptotically, as the dimension of the vector space becomes large, we give their exponential growth and determine their initial values. The initial values are expressed analytically in terms of theta functions and Euler's generating function for the partition numbers. Our asymptotic enumeration method is based on a Demazure module limit construction for integrable highest weight representations of affine Kac-Moody algebras. For the classical Galois numbers that count the number of subspaces in vector spaces over finite fields, the theta functions are Jacobi theta functions. We apply our findings to the asymptotic number of linear $q$-ary codes and conclude with some final remarks about possible future research concerning asymptotic enumerations via limit constructions for affine Kac-Moody algebras and modularity of characters of integrable highest weight representations.
\end{abstract}

\section{INTRODUCTION}

The generalized Galois numbers $G_{N}^{(r)}(q)$ count the number of flags $0=V_{0} \subseteq$ $V_{1} \subseteq \ldots \subseteq V_{r}=\mathbf{F}_{q}^{N}$ of length $r$ in an $N$-dimensional vector space over a field with $q$ elements [37. In particular, when $r=2$ these are the classical Galois numbers studied by Goldman and Rota [10] which give the total number of subspaces in $\mathbf{F}_{q}^{N}$.

We show that the generalized Galois numbers grow asymptotically, as $r, q$ are fixed and $N \rightarrow \infty$, exponentially with factor $O\left(N^{2}\right)$ in logarithmic "time" scale:

$$
G_{N}^{(r)}(q) \sim I_{r}(q) \cdot e^{O\left(N^{2}\right) \log (q)}
$$

Here, "time" equals the cardinality $q$ of the finite field. Our main result is the explicit analytic description of the initial values $I_{r}(q)$ via theta functions and Euler's generating function for the partition numbers. To our knowledge this is the first result that exhibits the asymptotic growth of Galois numbers in such generality and detail.

Received by the editors September 23, 2011 and, in revised form, December 7, 2011. 2010 Mathematics Subject Classification. Primary 05A16, 06B15; Secondary 94B05.

Key words and phrases. Asymptotic enumeration, Galois number, affine Kac-Moody algebra, Demazure module, linear code. 
This investigation serves three purposes. First, the generalized Galois numbers are of independent interest as they enumerate points in partial flag varieties, i.e. fundamental geometric objects defined over finite fields. For example, the classical Galois numbers

$$
G_{N}(q)=G_{N}^{(2)}(q)=\sum_{k=0}^{N}\left|\operatorname{Gr}(k, N)\left(\mathbf{F}_{q}\right)\right|
$$

count the number of $\mathbf{F}_{q}$-rational points in Grassmann varieties. The numbers of solutions of the set of equations for $\operatorname{Gr}(k, N)$ in extension fields $\mathbf{F}_{p^{n}}$ of $\mathbf{F}_{p}$ are subject to the study of local zeta-functions $Z(\operatorname{Gr}(k, N), t)=\exp \left(\sum_{n \geq 1}\left|\operatorname{Gr}(k, N)\left(\mathbf{F}_{p^{n}}\right)\right| \frac{t^{n}}{n}\right)$ in number theory. Let us mention that a generating function for the local zetafunction $Z(\operatorname{Gr}(k, N), t)$ can be given by

$$
Z(\operatorname{Gr}(k, N), t)=\frac{1}{(1-t)^{b_{0}}(1-p t)^{b_{1}} \ldots\left(1-p^{k(N-k)} t\right)^{b_{k(N-k)}}},
$$

where the $b_{i}=\operatorname{dim} H_{2 i}(\operatorname{Gr}(k, N)(\mathbf{C}), \mathbf{Z})$ are the even topological Betti numbers of the complex Grassmannian. Consequently, the study of Galois numbers reflects upon many subjects.

Second, the Galois numbers enumerate asymptotically the number of equivalence classes of linear $q$-ary codes in algebraic coding theory as recently shown by Hou and Wild [13, 14, 15, 39, 40. For example, the asymptotic number $N_{n, q}^{\mathfrak{S}}$ of linear $q$-ary codes under permutation equivalence is

$$
N_{n, q}^{\mathfrak{S}} \sim \frac{G_{n}(q)}{n !} .
$$

We apply our findings to those asymptotic equivalences and derive considerable simplifications of the asymptotic enumeration of linear $q$-ary codes (\$4).

Third, our investigation serves as the demonstration of the asymptotic enumeration method itself (\$3). We identify the generalized Galois numbers $G_{N}^{(r)}(q)$ as the so-called basic specialization of the Demazure modules $V_{-N \omega_{1}}\left(\Lambda_{0}\right)$ of the affine Kac-Moody algebra $\widehat{\mathfrak{s l}}_{r}$ (see (3.14)). Those characters pass via a graded limit construction [8, 23, 27, 28, to the characters of the fundamental representations of our affine Kac-Moody algebra:

$$
\lim _{n \rightarrow \infty} \chi\left(V_{-(r n+j) \omega_{1}}\left(\Lambda_{0}\right)\right)=\chi\left(V\left(\Lambda_{j}\right)\right) .
$$

By a symmetry argument, Kac's [17] character formula (see also [9, 35]) for the basic representation

$$
\chi\left(V\left(\Lambda_{0}\right)\right)=\sum_{k=0}^{\infty} p^{(r-1)}(k) e^{\Lambda_{0}-k \delta} \cdot \sum_{\gamma \in Q} e^{-\left(h\|\gamma\|^{2} \delta+\gamma\right)}
$$

then allows us to prove our main result:

Theorem 3.2. Consider the generalized Galois number $G_{N}^{(r)}(q)$. For any prime power $e^{\delta}=p^{m}$ (in fact, for any complex number $e^{\delta}$ where $\delta \in-2 \pi i \mathbf{H}$ ) and $0 \leq$ $j<r$ we have the limit

$$
\lim _{n \rightarrow \infty} G_{r n+j}^{(r)}\left(e^{\delta}\right) \cdot e^{-u_{j}(r, n) \delta}=\frac{\Theta_{F_{j}}\left(-\frac{\delta}{2 \pi i}\right)}{\phi\left(e^{-\delta}\right)^{r-1}} .
$$


Here, $\phi(x)^{-1}=\prod_{m=1}^{\infty}\left(1-x^{m}\right)^{-1}$ denotes Euler's generating function for the partition numbers and $\Theta_{F_{j}}(z)=\sum_{\mathbf{k} \in \mathbf{Z}^{r-1}} e^{2 \pi i z F_{j}(\mathbf{k})}$ are theta functions associated to the quadratic forms $F_{0}, F_{1}, \ldots, F_{r-1}$ on the lattice $\mathbf{Z}^{r-1}$ given by

$$
\begin{aligned}
& F_{0}\left(k_{1}, \ldots, k_{r-1}\right)=\sum_{l=1}^{r-1} k_{l}^{2}-\sum_{l=1}^{r-2} k_{l} k_{l+1}, \\
& F_{j}\left(k_{1}, \ldots, k_{r-1}\right)=\left(k_{j}+\frac{1}{2}\right)^{2}+\sum_{l=1, l \neq j}^{r-1} k_{l}^{2}-\sum_{l=1}^{r-2} k_{l} k_{l+1} .
\end{aligned}
$$

The exponents $u_{0}, u_{1}, \ldots, u_{r-1}$ are

$$
\begin{aligned}
& u_{0}(r, n)=\frac{r(r-1) n^{2}}{2}, \\
& u_{j}(r, n)=\frac{(r n+j)(r n+j-1)}{2}-\frac{r n(r n+2 j-r)}{2 r}+\frac{1}{4} .
\end{aligned}
$$

For the classical Galois numbers our theta functions turn out to be Jacobi theta functions (see Corollary 3.7), and we continue to study them in greater detail.

This asymptotic enumeration method is of general interest. To representation theorists, since our specializations of Demazure characters turn out to have important interpretations in combinatorial geometry, their limit constructions allow (analytic) solutions of asymptotic enumeration problems. To enumerative combinatorialists, our method yields an asymptotic enumeration framework in the following sense. Demazure characters are flexible in that they are parametrized by the Lie algebra type and rank, highest weights and Weyl group elements. Therefore, certain specializations of such characters could be employed to embed interesting combinatorial functions into this context. We will comment on immediate further generalizations of our asymptotic considerations along those parameters in $\$ 5$,

Let us conclude the introduction with the following remark on our asymptotic enumeration method. In the case of generalized Galois numbers we do not make use of the modularity of characters of integrable highest weight modules, since the prime powers $p^{-m}<1$ lie in the region of convergence of our modular forms. However, we will discuss in $\$ 5$ an important eventual application of our asymptotic enumeration method where modularity has to be exploited.

\section{Notation AND BACKGROUND}

The generalized Galois number $G_{N}^{(r)}(q) \in \mathbf{N}[q]$ can be defined as the specialization of the generalized $N$-th Rogers-Szegö polynomial at $(\mathbf{1}, q)$ [37:

$$
G_{N}^{(r)}(q)=H_{N}^{(r)}(\mathbf{1}, q)
$$

The $N$-th generalized Rogers-Szegö polynomial $H_{N}^{(r)}(\mathbf{z}, q) \in \mathbf{N}\left[z_{1}, \ldots, z_{r}, q\right]$ [29, 30, 36] (see [1] for an account) is defined as the generating function of the $q$-multinomial coefficients:

$$
H_{N}^{(r)}(\mathbf{z}, q)=\sum_{\substack{\mathbf{k}=\left(k_{1}, \ldots, k_{r}\right) \in \mathbf{N}^{r} \\
k_{1}+\ldots+k_{r}=N}}\left[\begin{array}{c}
N \\
\mathbf{k}
\end{array}\right]_{q} \mathbf{z}^{\mathbf{k}}
$$

Recall from [37] that the $q$-multinomial coefficient $\left[\begin{array}{c}N \\ k_{1}, \ldots, k_{r}\end{array}\right]_{q}$ counts the number of flags $0=V_{0} \subseteq \ldots \subseteq V_{r}=\mathbf{F}_{q}^{N}$ subject to the conditions $\operatorname{dim}\left(V_{i}\right)=k_{1}+\ldots+k_{i}$. 
For general facts about affine Kac-Moody algebras and their representation theory we refer the reader to [3, 18, and for Demazure modules to [8]. Let us briefly fix the notation we will use throughout. We consider the affine Kac-Moody algebra $\widehat{\mathfrak{s l}}_{r}$. We denote the simple roots by $\alpha_{0}, \alpha_{1}, \ldots, \alpha_{r-1}$, the highest root by $\theta=\alpha_{1}+\ldots+\alpha_{r-1}$ and the imaginary root by $\delta=\alpha_{0}+\theta$. The affine root lattice is then defined as $\widehat{Q}=\mathbf{Z} \alpha_{0} \oplus \mathbf{Z} \alpha_{1} \oplus \ldots \oplus \mathbf{Z} \alpha_{r-1}$, and the real span of the simple roots is given by $\widehat{\mathfrak{h}}_{\mathbf{R}}^{*}=\mathbf{R} \otimes_{\mathbf{z}} \widehat{Q}$. We have a non-degenerate symmetric bilinear form on $\widehat{\mathfrak{h}}_{\mathbf{R}}^{*}$ by $\left\langle\alpha_{i}, \alpha_{j}\right\rangle=c_{i j}$, where $C=\left(c_{i j}\right)$ is the Cartan matrix of $\widehat{\mathfrak{s l}}_{r}$, and we define $\|\cdot\|^{2}=(2 h)^{-1}\langle\cdot, \cdot\rangle$, where $h=r$ is the Coxeter number of $\widehat{\mathfrak{s l}}_{r}$. For a dominant integral weight $\Lambda=m_{1} \Lambda_{0}+m_{2} \Lambda_{1}+\ldots+m_{r-1} \Lambda_{r-1}$ in the affine weight lattice $\widehat{P}=\mathbf{Z} \Lambda_{0} \oplus \mathbf{Z} \Lambda_{1} \oplus \ldots \oplus \mathbf{Z} \Lambda_{r-1}$, we let $V(\Lambda)$ be the integrable highest weight representation of weight $\Lambda$ of $\widehat{\mathfrak{s l}}_{r}$ and $\chi(V(\Lambda))$ its character. The $\Lambda_{0}, \Lambda_{1}, \ldots, \Lambda_{r-1}$ are called fundamental weights, the $V\left(\Lambda_{l}\right)$ the fundamental representations and $V\left(\Lambda_{0}\right)$ the basic representation. As for the Demazure modules, we will consider only the translations $t_{-k \omega_{1}}=\left(s_{1} s_{2} \ldots s_{r-1} \sigma^{r-1}\right)^{k}$ in the extended affine Weyl group of $\widehat{\mathfrak{s l}}_{r}$, where $\omega_{1}=\Lambda_{1}-\Lambda_{0}$. Here, $\sigma$ denotes the automorphism of the Dynkin diagram of $\widehat{\mathfrak{s l}}_{r}$ which sends 0 to 1 , and $s_{1}, \ldots, s_{r-1}$ are the simple reflections associated to the simple roots $\alpha_{1}, \ldots, \alpha_{r-1}$. We denote the Demazure module associated to those translations by $V_{-k \omega_{1}}(\Lambda)$ and its character by $\chi\left(V_{-k \omega_{1}}(\Lambda)\right)$. We write the monomials in the characters of our modules as $e^{\lambda}$; the coefficient $k$ in the monomial $e^{-k \alpha_{0}}$ is referred to as the degree.

$\mathbf{H}$ will denote the upper half plane in $\mathbf{C}$. We write $\sim$ for asymptotic equivalence; that is, for $f, g: \mathbf{N} \rightarrow \mathbf{R}_{>0}$ we write $f(n) \sim g(n)$ if $\lim _{n \rightarrow \infty} f(n) / g(n)=1$.

\section{Asymptotics of Generalized Galois numbers}

We will start with a direct consequence of Kac's character formula [17, (3.37)] (see also [9, Proposition 2.1]).

Proposition 3.1. Consider the basic representation $V\left(\Lambda_{0}\right)$ of $\widehat{\mathfrak{s l}}_{r}$. Let $Q$ be the lattice $Q=\widehat{Q} / \mathbf{Z} \alpha_{0}=\mathbf{Z} \alpha_{1} \oplus \ldots \oplus \mathbf{Z} \alpha_{r-1} \cong \mathbf{Z}^{r-1}$. Then,

$$
\chi\left(V\left(\Lambda_{0}\right)\right)=\frac{e^{\Lambda_{0}}}{\phi\left(e^{-\delta}\right)^{r-1}} \cdot \sum_{\mathbf{k} \in \mathbf{Z}^{r-1}} e^{\frac{1}{4} \theta} \prod_{l=0}^{r-1} e^{-F_{l}(\mathbf{k}) \alpha_{l}} .
$$

Here, $\phi(x)^{-1}=\prod_{i=1}^{m}\left(1-x^{m}\right)^{-1}$ is Euler's generating function for the partition numbers, and the $F_{0}, F_{1}, \ldots, F_{r-1}$ are quadratic forms on the lattice $Q \cong \mathbf{Z}^{r-1}$ defined as

$$
\begin{aligned}
& F_{0}\left(k_{1}, \ldots, k_{r-1}\right)=\sum_{l=1}^{r-1} k_{l}^{2}-\sum_{l=1}^{r-2} k_{l} k_{l+1}, \\
& F_{j}\left(k_{1}, \ldots, k_{r-1}\right)=\left(k_{j}+\frac{1}{2}\right)^{2}+\sum_{l=1, l \neq j}^{r-1} k_{l}^{2}-\sum_{l=1}^{r-2} k_{l} k_{l+1} .
\end{aligned}
$$


Proof. Due to Kac [17, (3.37)] (see also [9, Proposition 2.1]) we have the following character formula for the basic representation $V\left(\Lambda_{0}\right)$ :

$$
\chi\left(V\left(\Lambda_{0}\right)\right)=\sum_{k=0}^{\infty} p^{(r-1)}(k) e^{\Lambda_{0}-k \delta} \cdot \sum_{\gamma \in Q} e^{-\left(h\|\gamma\|^{2} \delta+\gamma\right)} .
$$

The function $p^{(r-1)}(k)$ is defined via its generating function $\sum_{k=0}^{\infty} p^{(r-1)}(k) x^{k}=$ $\phi(x)^{-r+1}$. Let $f(\mathbf{k})$ be the quadratic form $f(\mathbf{k})=f\left(k_{1}, \ldots, k_{r-1}\right)=\sum_{i=1}^{r-1} k_{i}^{2}-$ $\sum_{i=1}^{r-2} k_{i} k_{i+1}$ on the lattice $Q \cong \mathbf{Z}^{r-1}$. If we express $\gamma \in Q$ as the linear combination $\gamma=k_{1} \alpha_{1}+\ldots+k_{r-1} \alpha_{r-1}$, we have $h\|\gamma\|^{2}=f(\mathbf{k})$. Then,

$$
\begin{aligned}
\chi\left(V\left(\Lambda_{0}\right)\right) & =\sum_{k=0}^{\infty} p^{(r-1)}(k) e^{\Lambda_{0}-k \delta} \cdot \sum_{\gamma \in Q} e^{-\left(h\|\gamma\|^{2} \delta+\gamma\right)} \\
& =\frac{e^{\Lambda_{0}}}{\phi\left(e^{-\delta}\right)^{r-1}} \cdot \sum_{\gamma \in Q} e^{-\left(h\|\gamma\|^{2} \delta+\gamma\right)} \\
& =\frac{e^{\Lambda_{0}}}{\phi\left(e^{-\delta}\right)^{r-1}} \cdot \sum_{\mathbf{k} \in \mathbf{Z}^{r-1}} e^{-f(\mathbf{k}) \delta} e^{-k_{1} \alpha_{1}} \ldots e^{-k_{r-1} \alpha_{r-1}} \\
& =\frac{e^{\Lambda_{0}}}{\phi\left(e^{-\delta}\right)^{r-1}} \cdot \sum_{\mathbf{k} \in \mathbf{Z}^{r-1}} e^{-f(\mathbf{k}) \alpha_{0}} e^{-\left(f(\mathbf{k})+k_{1}\right) \alpha_{1}} \ldots e^{-\left(f(\mathbf{k})+k_{r-1}\right) \alpha_{r-1}} .
\end{aligned}
$$

Note that $F_{0}(\mathbf{k})=f(\mathbf{k})$ and $F_{j}(\mathbf{k})-\frac{1}{4}=f(\mathbf{k})+k_{j}$. This finishes the proof.

We are ready to prove our main result.

Theorem 3.2. Consider the generalized Galois number $G_{N}^{(r)}(q)$. For any prime power $e^{\delta}=p^{m}$ (in fact, for any complex number $e^{\delta}$ where $\delta \in-2 \pi i \mathbf{H}$ ) and $0 \leq$ $j<r$ we have the limit

$$
\lim _{n \rightarrow \infty} G_{r n+j}^{(r)}\left(e^{\delta}\right) \cdot e^{-u_{j}(r, n) \delta}=\frac{\Theta_{F_{j}}\left(-\frac{\delta}{2 \pi i}\right)}{\phi\left(e^{-\delta}\right)^{r-1}} .
$$

Here, $\phi(x)^{-1}=\prod_{m=1}^{\infty}\left(1-x^{m}\right)^{-1}$ denotes Euler's generating function for the partition numbers, and $\Theta_{F_{j}}(z)=\sum_{\mathbf{k} \in \mathbf{Z}^{r-1}} e^{2 \pi i z F_{j}(\mathbf{k})}$ are theta functions associated to the quadratic forms $F_{0}, F_{1}, \ldots, F_{r-1}$ on the lattice $\mathbf{Z}^{r-1}$ given by

$$
\begin{aligned}
& F_{0}\left(k_{1}, \ldots, k_{r-1}\right)=\sum_{l=1}^{r-1} k_{l}^{2}-\sum_{l=1}^{r-2} k_{l} k_{l+1}, \\
& F_{j}\left(k_{1}, \ldots, k_{r-1}\right)=\left(k_{j}+\frac{1}{2}\right)^{2}+\sum_{l=1, l \neq j}^{r-1} k_{l}^{2}-\sum_{l=1}^{r-2} k_{l} k_{l+1} .
\end{aligned}
$$

The exponents $u_{0}, u_{1}, \ldots, u_{r-1}$ are

$$
\begin{aligned}
& u_{0}(r, n)=\frac{r(r-1) n^{2}}{2}, \\
& u_{j}(r, n)=\frac{(r n+j)(r n+j-1)}{2}-\frac{r n(r n+2 j-r)}{2 r}+\frac{1}{4} .
\end{aligned}
$$


Proof. Let $N=r n+j$ for $0 \leq j<r$ and consider the Demazure module $V_{-N \omega_{1}}\left(\Lambda_{0}\right)$ associated to the translation $t_{-N \omega_{1}}=\left(s_{1} s_{2} \ldots s_{r-1} \sigma^{r-1}\right)^{N}$. By Sanderson [31] we can describe its character $\chi\left(V_{-N \omega_{1}}\left(\Lambda_{0}\right)\right)$ via a certain specialization of a symmetric Macdonald polynomial (see [26, Chapter VI] for their definition and properties). That is, let $[N]=(N, 0, \ldots, 0) \in \mathbf{N}^{r}$ denote the one-row Young diagram and $\eta_{N}$ the smallest composition of degree $N$; i.e. since $N=r n+j$ we have $\eta_{N}=$ $\left((n)^{r-j},(n+1)^{j}\right) \in \mathbf{N}^{r}$. Following [31, §2] we have $[N]=t_{-N \omega_{1}} \cdot \eta_{N}$ with the convention $\sigma \cdot \eta_{N}=\eta_{N}$. Furthermore, by computing the expression $u([N])-u\left(\eta_{[N]}\right)$ in [31, Theorem 6], the maximal occurring degree in $\chi\left(V_{-N \omega_{1}}\left(\Lambda_{0}\right)\right)$ is given by

$$
d_{r}(N)=d_{r}(r n+j)=\frac{(r n+j)(r n+j-1)}{2}-\frac{r n(r n+2 j-r)}{2 r} .
$$

Note that $d_{r}(r n)=u_{0}(r, n)$ and $d_{r}(r n+j)=u_{j}(r, n)-\frac{1}{4}$ for $j=1, \ldots, r-1$. Let $\mathbf{z}=\left(e^{\Lambda_{1}-\Lambda_{0}}, e^{\Lambda_{2}-\Lambda_{1}}, \ldots, e^{\Lambda_{r-1}-\Lambda_{r-2}}, e^{\Lambda_{0}-\Lambda_{r-1}}\right)$. Then, by [31, Theorem 6 and 7$]$ we have

$$
\chi\left(V_{-N \omega_{1}}\left(\Lambda_{0}\right)\right)=e^{\Lambda_{0}-d_{r}(N) \delta} \cdot P_{[N]}\left(\mathbf{z} ; e^{\delta}, 0\right),
$$

where $P_{[N]}(\mathbf{z} ; q, 0)$ denotes the specialized symmetric Macdonald polynomial associated to the partition $[N]$. Furthermore, by Hikami [12, Equation (3.4)] this Macdonald polynomial equals the $N$-th generalized Rogers-Szegö polynomial:

$$
P_{[N]}(\mathbf{z} ; q, 0)=H_{N}^{(r)}(\mathbf{z}, q) .
$$

Combining (3.11) and (3.12) we obtain

$$
\chi\left(V_{-N \omega_{1}}\left(\Lambda_{0}\right)\right)=e^{\Lambda_{0}-d_{r}(N) \delta} \cdot H_{N}^{(r)}\left(\mathbf{z}, e^{\delta}\right) .
$$

Consequently, the basic specialization at $e^{-\alpha_{1}}=\ldots=e^{-\alpha_{r-1}}=1$ of the Demazure character on the left-hand side of $(3.13)$ gives the generalized Galois number $G_{N}^{(r)}$ up to translation:

$$
\left.\chi\left(V_{-N \omega_{1}}\left(\Lambda_{0}\right)\right)\right|_{\left(e^{-\alpha_{1}}=\ldots=e^{\left.-\alpha_{r-1}=1\right)}\right.}=e^{\Lambda_{0}-d_{r}(N) \delta} \cdot G_{N}^{(r)}\left(e^{\delta}\right) .
$$

Now, let us proceed to the limit considerations. By Fourier and Littelmann 8 , Theorem D] (which is based on work by Mathieu and Kumar [23, 27, 28]) the characters of our Demazure modules pass, as $N \rightarrow \infty$, as functions in $\left(e^{-\alpha_{0}}, e^{-\alpha_{1}}, \ldots\right.$, $\left.e^{-\alpha_{r-1}}\right)$ to the characters of the fundamental representations $V\left(\Lambda_{0}\right), V\left(\Lambda_{1}\right), \ldots$, $V\left(\Lambda_{r-1}\right)$ of $\widehat{\mathfrak{s l}}_{r}$ as follows:

$$
\lim _{n \rightarrow \infty} \chi\left(V_{-(r n+j) \omega_{1}}\left(\Lambda_{0}\right)\right)=\chi\left(V\left(\Lambda_{j}\right)\right) .
$$

We are ready to prove our claimed identity (3.5) in the case $j=0$. Recall that $\delta=\alpha_{0}+\theta$, where $\theta=\alpha_{1}+\ldots+\alpha_{r-1}$, and $d_{r}(r n)=u_{0}(r, n)$. Then, the equations

\footnotetext{
${ }^{1}$ There seems to be a misprint in [31 §4]. Namely, the image $\pi(q)$ should equal $q=e^{\delta}$, not $q=e^{-\delta}$.
} 
(3.14), (3.15) and the character formula (3.1) for the basic representation $V\left(\Lambda_{0}\right)$ imply

$$
\begin{aligned}
\lim _{n \rightarrow \infty} G_{r n}^{(r)}\left(e^{\delta}\right) \cdot e^{-u_{0}(r, n) \delta} & =\left.e^{-\Lambda_{0}} \cdot \chi\left(V\left(\Lambda_{0}\right)\right)\right|_{\left(e^{-\alpha_{1}}=\ldots=e^{-\alpha_{r-1}}=1\right)} \\
& =\frac{1}{\phi\left(e^{-\delta}\right)^{r-1}} \cdot \sum_{\mathbf{k} \in \mathbf{Z}^{r-1}} e^{-F_{0}(\mathbf{k}) \delta} \\
& =\frac{1}{\phi\left(e^{-\delta}\right)^{r-1}} \cdot \Theta_{F_{0}}\left(-\frac{\delta}{2 \pi i}\right) .
\end{aligned}
$$

There is a subtlety to our deduction in the cases $j=1, \ldots, r-1$. The characters of the representations $V\left(\Lambda_{0}\right), V\left(\Lambda_{1}\right), \ldots, V\left(\Lambda_{r-1}\right)$ are symmetrical in the sense that they subsequently differ by an application of the automorphism $\sigma$ that sends 0 to 1 in the Dynkin diagram of $\widehat{\mathfrak{s l}}_{r}$. Note that this $\sigma$ is a generator, i.e. a specific coset representative, of the cyclic group $\widehat{P} / \widehat{Q}$. An application of $\sigma$ cyclically shifts the fundamental weights $\Lambda_{i} \mapsto \Lambda_{i+1}$ and the simple roots $\alpha_{i} \mapsto \alpha_{i+1}$ (by cyclic we mean $\Lambda_{r}=\Lambda_{0}$ and $\left.\alpha_{r}=\alpha_{0}\right)$. Consequently, it leaves $\delta$ invariant $\sigma(\delta)=\delta$ and $\sigma^{j}(\theta)=\alpha_{0}+\theta-\alpha_{j}$ for $j=1, \ldots, r-1$. To be precise, Kac's character formula (3.1) for the fundamental representations $V\left(\Lambda_{1}\right), \ldots, V\left(\Lambda_{r-1}\right)$ reads as follows:

$$
\chi\left(V\left(\Lambda_{j}\right)\right)=\frac{e^{\Lambda_{j}}}{\phi\left(e^{-\delta}\right)^{r-1}} \cdot \sum_{\mathbf{k} \in \mathbf{Z}^{r-1}} e^{\frac{1}{4}\left(\alpha_{0}+\theta-\alpha_{j}\right)} \prod_{l=0}^{r-1} e^{-F_{l+r-j}(\mathbf{k}) \alpha_{l}} .
$$

Recall that $u_{j}(r, n)=d_{r}(r n+j)+\frac{1}{4}$. Therefore, we obtain

$$
\begin{aligned}
\lim _{n \rightarrow \infty} G_{r n+j}^{(r)}\left(e^{\delta}\right) \cdot e^{-u_{j}(r, n) \delta} & =e^{-\frac{1}{4} \delta} \cdot \lim _{n \rightarrow \infty} G_{r n+j}^{(r)}\left(e^{\delta}\right) \cdot e^{-d_{r}(r n+j) \delta} \\
& =\left.e^{-\frac{1}{4} \delta} \cdot e^{-\Lambda_{j}} \cdot \chi\left(V\left(\Lambda_{j}\right)\right)\right|_{\left(e^{-\alpha_{1}}=\ldots=e^{-\alpha_{r-1}}=1\right)} \\
& =\frac{e^{-\frac{1}{4} \delta}}{\phi\left(e^{-\delta}\right)^{r-1}} \cdot \sum_{\mathbf{k} \in \mathbf{Z}^{r-1}} e^{\frac{1}{4} \delta} e^{-F_{j}(\mathbf{k}) \delta} \\
& =\frac{1}{\phi\left(e^{-\delta}\right)^{r-1}} \cdot \Theta_{F_{j}}\left(-\frac{\delta}{2 \pi i}\right) .
\end{aligned}
$$

This establishes the theorem.

Remark 3.3. Motivated by Kac's character formula (3.4) we write the quadratic forms on $Q=\mathbf{Z} \alpha_{1} \oplus \ldots \oplus \mathbf{Z} \alpha_{r-1} \cong \mathbf{Z}^{r}$ intrinsically in terms of data associated to our affine Kac-Moody algebra $\widehat{\mathfrak{s l}}_{r}$ as follows. For $j=1, \ldots, r-1$ one has

$$
\begin{aligned}
& F_{0}(\gamma)=h\|\gamma\|^{2} \\
& F_{j}(\gamma)=h\|\gamma\|^{2}+\left\langle\Lambda_{j}, \gamma\right\rangle+\frac{1}{4} .
\end{aligned}
$$

The exponents $u_{0}, u_{1}, \ldots, u_{r-1}$ can be described via the translation formula [18, (6.5.3)].

Remark 3.4. One can phrase Theorem 3.2 asymptotically as

$$
G_{r n+j}^{(r)}\left(e^{\delta}\right) \sim \frac{\Theta_{F_{j}}\left(-\frac{\delta}{2 \pi i}\right)}{\phi\left(e^{-\delta}\right)^{r-1}} \cdot\left(e^{\delta}\right)^{u_{j}(n, r)} .
$$

Note that for fixed $r$ the exponents $u_{j}(n, r)$ lie in $O\left(n^{2}\right)$. 
Remark 3.5. For $j=1, \ldots, r-1$ the limits in Theorem 3.2 coincide. In fact, the quadratic forms $F_{1}, \ldots, F_{r-1}$ differ only by a cyclic shift of the coordinates. Summation over the complete lattice $\mathbf{Z}^{r-1}$ produces equality.

Remark 3.6. In order to obtain our limiting expressions, we "only" make use of Kac's explicit character formula (3.4). However, we would like to refer the reader to the explicit Frenkel-Kac-Segal vertex operator construction [9, 35] of the basic representation, which must be taken into account for future considerations concerning asymptotics of Galois-type numbers.

Let us summarize the implications of Theorem 3.2 for the classical Galois numbers $G_{N}(q)=G_{N}^{(2)}(q)$ that count the number of subspaces in $\mathbf{F}_{q}^{N}$.

Corollary 3.7. Consider the classical Galois numbers $G_{N}(q)$. For any prime power $q=p^{m}$ (in fact, for any complex number $|q|>1$ ) we have

$$
\begin{gathered}
g_{2 \infty+1}(q)=\lim _{n \rightarrow \infty} G_{2 n+1}(q) \cdot q^{-\frac{(2 n+1)^{2}}{4}}=\frac{\vartheta_{2}\left(0, q^{-1}\right)}{\phi\left(q^{-1}\right)}, \\
g_{2 \infty}(q)=\lim _{n \rightarrow \infty} G_{2 n}(q) \cdot q^{-\frac{(2 n)^{2}}{4}}=\frac{\vartheta_{3}\left(0, q^{-1}\right)}{\phi\left(q^{-1}\right)} .
\end{gathered}
$$

Here, $\phi(x)^{-1}=\prod_{m=1}^{\infty}\left(1-x^{m}\right)^{-1}$ denotes Euler's generating function for the partition numbers, and $\vartheta_{2}, \vartheta_{3}$ are the Jacobi theta functions

$$
\begin{aligned}
& \vartheta_{2}(z, q)=\sum_{k=-\infty}^{\infty} q^{\left(k+\frac{1}{2}\right)^{2}} e^{(2 k+1) i z}, \\
& \vartheta_{3}(z, q)=\sum_{k=-\infty}^{\infty} q^{k^{2}} e^{2 k i z} .
\end{aligned}
$$

The limits differ by

$$
g_{2 \infty}(q)-g_{2 \infty+1}(q)=\frac{\vartheta_{4}\left(0, q^{-\frac{1}{4}}\right)}{\phi\left(q^{-1}\right)},
$$

where $\vartheta_{4}$ is the Jacobi theta function

$$
\vartheta_{4}(z, q)=\sum_{k=-\infty}^{\infty}(-1)^{k} q^{k^{2}} e^{2 k i z} .
$$

Proof. It remains to prove (3.22). This follows from the identity

$$
\vartheta_{4}(z, q)=\vartheta_{3}\left(2 z, q^{4}\right)-\vartheta_{2}\left(2 z, q^{4}\right)
$$

[38, p. 464].

For completeness, we take a closer look at the numbers $g_{2 \infty+1}(q), g_{2 \infty}(q)$ and their differences.

Corollary 3.8. If $q$ is a prime power (in fact, $q \geq 2$ ), then

$$
g_{2 \infty}(2) \geq g_{2 \infty}(q)>g_{2 \infty+1}(q)>0 .
$$

For large values of $q$ we have

$$
\begin{aligned}
g_{2 \infty+1}(q) \cdot q^{\frac{(2 n+1)^{2}}{4}} & \sim 2 q^{n(n-1)}, \\
g_{2 \infty}(q) \cdot q^{n^{2}} & \sim q^{n^{2}}
\end{aligned}
$$


and consequently

$$
\begin{array}{r}
\lim _{q \rightarrow \infty} g_{2 \infty+1}(q)=0, \\
\lim _{q \rightarrow \infty} g_{2 \infty}(q)=1 .
\end{array}
$$

Proof. Our statements can be deduced from the Jacobi triple product identity. Namely, we have $\vartheta_{4}\left(0, q^{-1}\right)=\prod_{m=1}^{\infty}\left(1-q^{-2 m}\right)\left(1-q^{-(2 m-1)}\right)^{2}$, which is $>0$ if $q>1$. Since $\phi\left(q^{-1}\right)>0$ for $q>1$, the strict inequality $g_{2 \infty}(q)>g_{2 \infty+1}(q)$ is established. For the obviously sharp bound $g_{2 \infty}(2) \geq g_{2 \infty}(q)$ we look at

$$
\begin{aligned}
\frac{\vartheta_{3}\left(0, q^{-1}\right)}{\phi\left(q^{-1}\right)} & =\frac{\prod_{m=1}^{\infty}\left(1-q^{-2 m}\right)\left(1+q^{-(2 m-1)}\right)^{2}}{\prod_{m=1}^{\infty}\left(1-q^{-m}\right)} \\
& =\prod_{m=1}^{\infty}\left(1+q^{-m}\right)\left(1+q^{-(2 m-1)}\right)^{2},
\end{aligned}
$$

which is a product of compositions of monotonic functions on $q>1$. This identity also shows (3.25) and (3.27). To prove $g_{2 \infty+1}(q)>0$, (3.24) and (3.26), one considers

$$
\begin{aligned}
\frac{\vartheta_{2}\left(0, q^{-1}\right)}{\phi\left(q^{-1}\right)} & =\frac{2 q^{-\frac{1}{4}} \prod_{m=1}^{\infty}\left(1-q^{-2 m}\right)\left(1+q^{-2 m}\right)^{2}}{\prod_{m=1}^{\infty}\left(1-q^{-m}\right)} \\
& =2 q^{-\frac{1}{4}} \prod_{m=1}^{\infty}\left(1+q^{-m}\right)\left(1+q^{-2 m}\right)^{2}
\end{aligned}
$$

For some prime powers $q$, the numbers $g_{2 \infty+1}(q), g_{2 \infty}(q)$ and their differences have been listed in Table 1. The table has been produced in Mathematica with the following functions (up to a 10 digit precision: $\mathrm{N}[, 10]$ ) for $g_{2 \infty+1}(q), g_{2 \infty}(q)$ and $g_{2 \infty}(q)-g_{2 \infty+1}(q)$ respectively:

$$
\begin{aligned}
& f\left[q_{-}\right]:=N[E l l i p t i c T h e t a[2,0,1 / q] 1 / \text { QPochhammer }[1 / q, 1 / q], 10] \\
& g\left[q_{-}\right]:=N[E l l i p t i c T h e t a[3,0,1 / q] 1 / \text { Pochhammer }[1 / q, 1 / q], 10] \\
& h\left[q_{-}\right]:=N\left[E l l i p t i c T h e t a\left[4,0, q^{-}(-1 / 4)\right] 1 / \text { Pochhammer }[1 / q, 1 / q], 10\right]
\end{aligned}
$$

Certainly, our Theorem 3.2 allows an implementation for evaluating the asymptotic initial values of generalized Galois numbers.

Remark 3.9. Corollary 3.7 can be derived by the character formula of Feingold and Lepowsky [6, Theorem 4.5] for the basic representation of $\widehat{\mathfrak{s l}}_{2}$. That is,

$$
\chi\left(V\left(\Lambda_{0}\right)\right)=\sum_{k=0}^{\infty} p(k) e^{\Lambda_{0}-k \delta} \sum_{l=-\infty}^{\infty} e^{-l^{2} \alpha_{0}} e^{-l(l+1) \alpha_{1}},
$$

where $p(k)$ is the partition function that counts the number of ways to write $k$ as a sum of positive integers. In fact, Kac's character formula [17, (3.37)] reduces to this expression (see [17, (3.39)]), and our proof of Theorem 3.2 reduces to this setting. 
TABle 1. Asymptotic Galois numbers

$\begin{array}{cccc}q & g_{2 \infty+1}(q) & g_{2 \infty}(q) & g_{2 \infty}(q)-g_{2 \infty+1}(q) \\ & & & \\ 3 & 7.371949491 & 7.371968801 & 0.0000193107 \\ 5 & 3.018269046 & 3.019783846 & 0.0015147993 \\ 7 & 1.829548122 & 1.845509008 & 0.0159608865 \\ 11 & 1.499386995 & 1.537469387 & 0.0380823915 \\ 13 & 1.229171217 & 1.312069129 & 0.0828979124 \\ 17 & 1.155207999 & 1.258137150 & 0.1029291515 \\ 19 & 1.054013475 & 1.191906557 & 0.1378930825 \\ 23 & 0.95847868871 & 1.138621162 & 0.1801424752 \\ 29 & 0.8947912163 & 1.108510891 & 0.2137196747 \\ 29^{2011} & 1.203473556 \cdot 10^{-735} & 1.000000000 & 1.0000000000\end{array}$

\section{Applichtions to linear codes}

To describe the asymptotic number of non-equivalent binary $n$-codes in terms of the classical Galois numbers $G_{n}(2)$, Wild [39, 40] examines numbers $d_{1}(q), d_{2}(q)$ (see Lemma 1 in both articles) which, in the notation of Corollary 3.7, are defined as

$$
\begin{aligned}
& d_{1}(q)=g_{2 \infty+1}(q), \\
& d_{2}(q)=g_{2 \infty}(q) .
\end{aligned}
$$

He proves that they are positive constants (depending on $q$ ) less than 32 , gives a numerical evaluation method by use of the recursion formula of Goldman and Rota [10, (5)], evaluates $d_{1}(q), d_{2}(q)$ numerically for $q=2$, and shows $d_{1}(q)<d_{2}(q)$ for general $q$. Now, the detailed analytic behavior of those numbers can be extracted from Corollary 3.7 and Corollary 3.8 (see also Table 1 for examples).

For a general prime power $q$, Hou [13, 15] derives asymptotic equivalences for the numbers of linear $q$-ary codes under three notions of equivalence, that is, the permutation equivalence $(\mathfrak{S})$, the monomial equivalence $(\mathfrak{M})$, and semi-linear monomial equivalence $(\Gamma)$. He proves

$$
\begin{aligned}
N_{n, q}^{\mathfrak{S}} & \sim \frac{G_{n}(q)}{n !}, \\
N_{n, q}^{\mathfrak{M}} & \sim \frac{G_{n}(q)}{n !(q-1)^{n-1}}, \\
N_{n, q}^{\Gamma} & \sim \frac{G_{n}(q)}{n !(q-1)^{n-1} a},
\end{aligned}
$$

where $a=\left|\operatorname{Aut}\left(\mathbf{F}_{q}\right)\right|=\log _{p}(q)$ with $p=\operatorname{char}\left(\mathbf{F}_{q}\right)$. The asymptotic equivalence $N_{n, 2}^{\mathfrak{S}} \sim \frac{G_{n}(2)}{n !}$ concerns binary codes and was previously derived by Wild [39, 40]. Based on their results, the transitivity of $\sim$ and our Corollary 3.7 produce the following list. 
Corollary 4.1. The asymptotic numbers of linear q-ary codes, as $q$ is fixed and $n \rightarrow \infty$, under the three notions of equivalence $(\mathfrak{S})$, (M) and $(\Gamma)$ are given by

$$
\begin{aligned}
N_{2 n+1, q}^{\mathfrak{S}} & \sim \frac{\vartheta_{2}\left(0, q^{-1}\right)}{\phi\left(q^{-1}\right)} \cdot \frac{q^{\frac{(2 n+1)^{2}}{4}}}{(2 n+1) !}, \\
N_{2 n, q}^{\mathfrak{S}} & \sim \frac{\vartheta_{3}\left(0, q^{-1}\right)}{\phi\left(q^{-1}\right)} \cdot \frac{q^{n^{2}}}{(2 n) !}, \\
N_{2 n+1, q}^{\mathfrak{M}} & \sim \frac{\vartheta_{2}\left(0, q^{-1}\right)}{\phi\left(q^{-1}\right)} \cdot \frac{q^{\frac{(2 n+1)^{2}}{4}}}{(2 n+1) !(q-1)^{2 n}}, \\
N_{2 n, q}^{\mathfrak{M}} & \sim \frac{\vartheta_{3}\left(0, q^{-1}\right)}{\phi\left(q^{-1}\right)} \cdot \frac{q^{n^{2}}}{(2 n) !(q-1)^{2 n-1}}, \\
N_{2 n+1, q}^{\Gamma} & \sim \frac{\vartheta_{2}\left(0, q^{-1}\right)}{\phi\left(q^{-1}\right)} \cdot \frac{q^{\frac{(2 n+1)^{2}}{4}}}{(2 n+1) !(q-1)^{2 n} a}, \\
N_{2 n, q}^{\Gamma} & \sim \frac{\vartheta_{3}\left(0, q^{-1}\right)}{\phi\left(q^{-1}\right)} \cdot \frac{q^{n^{2}}}{(2 n) !(q-1)^{2 n-1} a} .
\end{aligned}
$$

Furthermore, for large prime powers $q$ one has

$$
\begin{aligned}
\frac{\vartheta_{2}\left(0, q^{-1}\right)}{\phi\left(q^{-1}\right)} \cdot q^{\frac{(2 n+1)^{2}}{4}} & \sim 2 q^{n(n-1)}, \\
\frac{\vartheta_{3}\left(0, q^{-1}\right)}{\phi\left(q^{-1}\right)} \cdot q^{n^{2}} & \sim q^{n^{2}} .
\end{aligned}
$$

For the last two statements see Corollary 3.8

\section{Conclusion}

The asymptotic enumeration method presented in this article can be summarized as follows. Once a certain specialization of Demazure characters has been identified with an interesting combinatorial function, the limit construction for affine Kac-Moody algebras can be used to carry it along towards the character of the integrable highest weight module and to derive asymptotic identities. There are at least two bottlenecks that one has to pass. First, a suitable character formula (for the limiting integrable highest weight representation) that performs well with the chosen specialization has to be available. Fortunately, there are a great number of results and literature available, e.g. [6, 17, 19, 20] (see also [2, 7, 21]). Second, the domain of the combinatorial function that enumerates the objects in question must lie in the region of convergence of the limiting expressions. For example, Demazure modules specialize to tensor products of representations of the underlying finite-dimensional Lie algebra. Unfortunately, the analytic string functions limiting the tensor product multiplicities cannot be simply evaluated, for reasons of (non)convergence, at the value 1 . A much finer analysis of their asymptotic behavior when $q \rightarrow 1$ is needed, one that has to exploit the fact that we deal with modular forms [20]. Such an asymptotic analysis must take the maximal weights in the integrable highest weight module into account where those string functions emerge, possibly borrowing and mimicking terminology from stochastic analysis such as the central limit region, the moderate and strong deviations region, and the region of 
rare events. An investigation of tensor product multiplicities along those lines is planned in a future publication.

An immediate generalization of our asymptotic considerations is to investigate the simply-laced-type analogs of generalized Galois numbers, that is, basic specializations of Demazure modules limited by the basic modules for affine Lie algebras of types $D$ and $E$. This involves first establishing the proper geometric interpretation of the basic specialization of such Demazure modules in terms of counting points in varieties defined over finite fields. The starting point of such considerations should be Ion's article [16], which generalizes Sanderson's 31] connection between Macdonald polynomials and Demazure characters from type $A$ to the simply-laced cases. Parallel to Ion [16] one should consider Kuniba et al. 25, which contains explicit formulas for Demazure characters in terms of $q$-multinomial coefficients which should serve the geometric interpretation. Such efforts could be part of a more general discussion of the higher level module cases, which amounts to the study of $q$-supernomials $11,22,32,33,34$ that generalize $q$-multinomials. One should possibly start with the simplest case of higher level (Demazure) modules for type $A_{1}^{(1)}$. See [4, 5] for higher level Demazure modules in this setting, and start e.g. with [6, $\S 5, \S 6]$ for explicit character formulas of higher level modules of

type $A_{1}^{(1)}$. These discussions, in particular the higher level situation including types different from $A$, are also subject to a future publication.

An interesting alternative project could be to re-interpret our asymptotic enumeration method geometrically through the geometric realization of Demazure and integrable highest weight modules via cohomology of Schubert and flag varieties [24].

\section{ACKNOWLEDGEMENTS}

This work was supported by the Swiss National Science Foundation (grant PP00P2-128455), the National Centre of Competence in Research 'Quantum Science and Technology', the German Science Foundation (SFB/TR12, and grants CH 843/1-1, CH 843/2-1), and the Excellence Initiative of the German Federal and State Governments through the Junior Research Group Program within the Institutional Strategy ZUK 43.

The author would like to thank Matthias Christandl for his kind hospitality at the ETH Zurich; Peter Littelmann, who drew his attention towards the Demazure module limit construction a couple of years ago; Thomas Bliem, who pointed him towards Rogers-Szegö polynomials; Ghislain Fourier for many helpful conversations; and the anonymous referee for detailed and valuable comments and suggestions that improved the knowledge of the author and the quality of the present article.

\section{REFERENCES}

1. George Andrews, The theory of partitions, Cambridge Mathematical Library, Cambridge University Press, Cambridge, 1998. Reprint of the 1976 original. MR.1634067 (99c:11126)

2. Kathrin Bringmann and Ken Ono, Some characters of Kac and Wakimoto and nonholomorphic modular functions, Math. Ann. 345 (2009), no. 3, 547-558. MR2534107 (2010f:11061)

3. Roger Carter, Lie algebras of finite and affine type, Cambridge Studies in Advanced Mathematics, vol. 96, Cambridge University Press, Cambridge, 2005. MR2188930 (2006i:17001)

4. Boris Feigin and Evgeny Feigin, $Q$-characters of the tensor products in $\mathfrak{s l}_{2}$-case, Mosc. Math. J. 2 (2002), no. 3, 567-588. MR.1988973 (2004e:17002) 
5. Integrable $\widehat{\mathfrak{s l}}_{2}$-modules as infinite tensor products, Fundamental mathematics today (Russian), Nezavis. Mosk. Univ., Moscow, 2003, pp. 304-334. MR2072647 (2005j:17007)

6. Alex Feingold and James Lepowsky, The Weyl-Kac character formula and power series identities, Adv. in Math. 29 (1978), no. 3, 271-309. MR.509801(83a:17015)

7. Amanda Folsom, Kac-Wakimoto characters and universal mock theta functions, Trans. Amer. Math. Soc. 363 (2011), no. 1, 439-455. MR2719689(2011h:11044)

8. Ghislain Fourier and Peter Littelmann, Weyl modules, Demazure modules, KR-modules, crystals, fusion products and limit constructions, Adv. Math. 211 (2007), no. 2, 566-593. MR2323538 (2008k:17005)

9. Igor Frenkel and Victor Kac, Basic representations of affine Lie algebras and dual resonance models, Invent. Math. 62 (1980/81), no. 1, 23-66. MR595581 (84f:17004)

10. Jay Goldman and Gian-Carlo Rota, The number of subspaces of a vector space, Recent Progress in Combinatorics (Proc. Third Waterloo Conf. on Combinatorics, 1968), Academic Press, New York, 1969, pp. 75-83. MR0252232 (40:5453)

11. Goro Hatayama, Anatol Kirillov, Atsuo Kuniba, Masato Okado, Taichiro Takagi, and Yasuhiko Yamada, Character formulae of $\widehat{\mathfrak{s l}}_{n}$-modules and inhomogeneous paths, Nuclear Phys. B 536 (1999), no. 3, 575-616. MR.1663325 (2000c:17022)

12. Kazuhiro Hikami, Representation of the Yangian invariant motif and the Macdonald polynomial, J. Phys. A 30 (1997), no. 7, 2447-2456. MR1457389(98j:81153)

13. Xiang-Dong Hou, On the asymptotic number of non-equivalent q-ary linear codes, J. Combin. Theory Ser. A 112 (2005), no. 2, 337-346. MR2177491 (2006h:94252)

14. On the asymptotic number of non-equivalent binary linear codes, Finite Fields Appl. 13 (2007), no. 2, 318-326. MR2307131 (2008c:94042)

15. _ Asymptotic numbers of non-equivalent codes in three notions of equivalence, Linear Multilinear Algebra 57 (2009), no. 2, 111-122. MR2492098(2010b:94080)

16. Bogdan Ion, Nonsymmetric Macdonald polynomials and Demazure characters, Duke Math. J. 116 (2003), no. 2, 299-318. MR1953294(2004d:33019)

17. Victor Kac, Infinite-dimensional algebras, Dedekind's $\eta$-function, classical Möbius function and the very strange formula, Adv. in Math. 30 (1978), no. 2, 85-136. MR513845 (83a:17014a)

18. _ Infinite-dimensional Lie algebras, third ed., Cambridge University Press, 1990. MR1104219 (92k:17038)

19. Victor Kac and Dale Peterson, Affine Lie algebras and Hecke modular forms, Bull. Amer. Math. Soc. (N.S.) 3 (1980), no. 3, 1057-1061. MR585190 (82b:10028)

20. Infinite-dimensional Lie algebras, theta functions and modular forms, Adv. in Math. 53 (1984), no. 2, 125-264. MR750341 (86a:17007)

21. Victor Kac and Minoru Wakimoto, Integrable highest weight modules over affine superalgebras and Appell's function, Comm. Math. Phys. 215 (2001), no. 3, 631-682. MR.1810948 (2001j:17017)

22. Anatol Kirillov, New combinatorial formula for modified Hall-Littlewood polynomials, $q$-series from a contemporary perspective (South Hadley, MA, 1998), Contemp. Math., vol. 254, Amer. Math. Soc., Providence, RI, 2000, pp. 283-333. MR1768934 (2001h:05103)

23. Shrawan Kumar, Demazure character formula in arbitrary Kac-Moody setting, Invent. Math. 89 (1987), no. 2, 395-423. MR894387 (88i:17018)

24. __ Kac-Moody groups, their flag varieties and representation theory, Progress in Mathematics, vol. 204, Birkhäuser Boston Inc., Boston, MA, 2002. MR.1923198 (2003k:22022)

25. Atsuo Kuniba, Kailash Misra, Masato Okado, Taichiro Takagi, and Jun Uchiyama, Characters of Demazure modules and solvable lattice models, Nuclear Phys. B 510 (1998), no. 3, 555-576. MR1607188 (99m:17019)

26. Ian Macdonald, Symmetric functions and Hall polynomials, second ed., Oxford Mathematical Monographs, The Clarendon Press, Oxford University Press, New York, 1995. With contributions by A. Zelevinsky, Oxford Science Publications. MR1354144 (96h:05207)

27. Olivier Mathieu, Construction du groupe de Kac-Moody et applications, C. R. Acad. Sci. Paris Sér. I Math. 306 (1988), no. 5, 227-230. MR932325(89e:17013)

28. __ Formules de caractères pour les algèbres de Kac-Moody générales, Astérisque (1988), no. 159-160, 267. MR980506 (90d:17024)

29. Leonard Rogers, On a three-fold symmetry in the elements of Heine's series, Proc. Lond. Math. Soc. 24 (1893), 171-179. 
30. - On the expansion of certain infinite products, Proc. Lond. Math. Soc. 24 (1893), 337-352.

31. Yasmine Sanderson, On the connection between Macdonald polynomials and Demazure characters, J. Algebraic Combin. 11 (2000), no. 3, 269-275. MR1771615(2001h:17018)

32. Anne Schilling, q-supernomial coefficients: from riggings to ribbons, MathPhys Odyssey, 2001, Prog. Math. Phys., vol. 23, Birkhäuser Boston, Boston, MA, 2002, pp. 437-454. MR.1903985 (2003e:05146)

33. Anne Schilling and Ole Warnaar, Supernomial coefficients, polynomial identities and q-series, Ramanujan J. 2 (1998), no. 4, 459-494. MR.1665322 (2000c:33023)

34. Inhomogeneous lattice paths, generalized Kostka polynomials and $A_{n-1}$ supernomials, Comm. Math. Phys. 202 (1999), no. 2, 359-401. MR1690046 (2000m:05234)

35. Graeme Segal, Unitary representations of some infinite-dimensional groups, Comm. Math. Phys. 80 (1981), no. 3, 301-342. MR626704 (82k:22004)

36. Gábor Szegő, Ein Beitrag zur Theorie der Thetafunktionen, S.B. Preuss. Akad. Wiss. Phys.Math. KI. (1926), 242-252.

37. Ryan Vinroot, Multivariate Rogers-Szegö polynomials and flags in finite vector spaces (2010), arXiv:1011.0984.

38. Edmund Whittaker and George Watson, A course of modern analysis, fourth edition. Reprinted, Cambridge University Press, New York, 1962. MR0178117 (31:2375)

39. Marcel Wild, The asymptotic number of inequivalent binary codes and nonisomorphic binary matroids, Finite Fields Appl. 6 (2000), no. 2, 192-202. MR1755766 (2001i:94077)

40. - The asymptotic number of binary codes and binary matroids, SIAM J. Discrete Math. 19 (2005), no. 3, 691-699 (electronic). MR2191288 (2006h:94049)

Institute for Theoretical Physics, ETH Zürich, Wolfgang-Pauli-Strasse 27, CH8093 Zürich, Switzerland - And - Institute of Physics, University of Freiburg, RheinStrasse 10, 79104 Freiburg, Germany

Current address: Schenkendorfstr. 11, 53173 Bonn, Germany

E-mail address: st.kousidis@googlemail.com 